\title{
Genetic Epidemiology and Clinical Features of Hereditary Hearing Impairment in the Taiwanese Population
}

\author{
Chen-Chi Wu ${ }^{1}$, Cheng-Yu Tsai ${ }^{1,2}{ }^{,}$Yi-Hsin Lin ${ }^{1}$, Pey-Yu Chen ${ }^{3}{ }^{\mathbb{D}}$, Pei-Hsuan Lin ${ }^{4}$, \\ Yen-Fu Cheng ${ }^{5,6}{ }^{\circ}$, Che-Ming $\mathrm{Wu}^{7}{ }^{7}$, Yin-Hung Lin ${ }^{2}$, Chee-Yee Lee ${ }^{8}$, \\ Jargalkhuu Erdenechuluun 9,10,11, Tien-Chen Liu ${ }^{1}(\mathbb{D})$, Pei-Lung Chen ${ }^{2,12, *}$ and \\ Chuan-Jen $\mathrm{Hsu}^{1,8}$ (D) \\ 1 Department of Otolaryngology, National Taiwan University Hospital, Taipei 10002, Taiwan; \\ jimchenchiwu@gmail.com (C.-C.W.); leon9139@gmail.com (C.-Y.T.); e314032@gmail.com (Y.-H.L.); \\ liuent@ntu.edu.tw (T.-C.L.); cjhsu@ntu.edu.tw (C.-J.H.) \\ 2 Graduate Institute of Medical Genomics and Proteomics, National Taiwan University College of Medicine, \\ Taipei 10055, Taiwan; yinhunglin@gmail.com \\ 3 Department of Otolaryngology, Mackay Memorial Hospital, Taipei 10449, Taiwan; lactopiscis@gmail.com \\ 4 Department of Otolaryngology, National Taiwan University Hospital Yunlin Branch, Yunlin 64041, Taiwan; \\ ru3au3@gmail.com \\ 5 Department of Medical Research, Taipei Veterans General Hospital, Taipei 11217, Taiwan; \\ yfcheng2@vghtpe.gov.tw \\ 6 Department of Otolaryngology-Head and Neck Surgery, Taipei Veterans General Hospital, \\ Taipei 11217, Taiwan \\ 7 Department of Otolaryngology-Head and Neck Surgery, Chang Gung Memorial Hospital, \\ Chang Gung University, Linkou 33302, Taiwan; bobwu506@hotmail.com \\ 8 Department of Otolaryngology, Buddhist Tzuchi General Hospital, Taichung Branch, \\ Taichung 42743, Taiwan; allenlee0919@gmail.com \\ 9 Department of Otolaryngology, Mongolian National University of Medical Sciences, \\ Ulaanbaatar 14210, Mongolia; emjj_jack@yahoo.com \\ 10 The EMJJ Otolaryngology Hospital, Ulaanbaatar 14210, Mongolia \\ 11 Department of Otolaryngology, National Center for Maternal and Child Health, \\ Ulaanbaatar 16060, Mongolia \\ 12 Department of Medical Genetics, National Taiwan University Hospital, Taipei 10041, Taiwan \\ * Correspondence: paylong@ntu.edu.tw
}

Received: 1 August 2019; Accepted: 27 September 2019; Published: 1 October 2019

check for updates

\begin{abstract}
Hereditary hearing impairment (HHI) is a common but heterogeneous clinical entity caused by mutations in a plethora of deafness genes. Research over the past few decades has shown that the genetic epidemiology of HHI varies significantly across populations. In this study, we used different genetic examination strategies to address the genetic causes of HHI in a large Taiwanese cohort composed of $>5000$ hearing-impaired families. We also analyzed the clinical features associated with specific genetic mutations. Our results demonstrated that next-generation sequencing-based examination strategies could achieve genetic diagnosis in approximately half of the families. Common deafness-associated genes in the Taiwanese patients assessed, in the order of prevalence, included GJB2, SLC26A4, OTOF, MYO15A, and MTRNR1, which were similar to those found in other populations. However, the Taiwanese patients had some unique mutations in these genes. These findings may have important clinical implications for refining molecular diagnostics, facilitating genetic counseling, and enabling precision medicine for the management of HHI.
\end{abstract}


Keywords: next-generation sequencing; population genomics; deafness; genetic diagnosis; genetic examination; precision medicine

\section{Introduction}

Hearing impairment is the most common inherited sensory defect. It is estimated that permanent sensorineural hearing impairment (SNHI) occurs in approximately 1.9 out of 1000 live births [1], and including late-onset SNHI, the disorder may affect $2 \%$ of school-age children [2,3]. About one-half to two-thirds of SNHI cases in children are attributed to genetic causes and are classified as hereditary hearing impairment (HHI) [4]. To date, more than 100 genes have been identified as causally related to HHI (http://hereditaryhearingloss.org). Because different genetic mutations represent different pathogeneses and are related to different clinical outcomes, identifying etiology is crucial in the assessment and management of childhood SNHI.

Over the past two decades, research has revealed that the genetic epidemiology of HHI varies across populations. For instance, c.235delC, c.35delG, and c.167delT are the most common GJB2 mutations in East Asians [5-7], Caucasians [8-11], and Ashkenazi Jews [12], respectively, whereas the c.-23+1G $>$ A mutation is unique to Southwest and South Asians [13-16]. Similarly, predominant SLC26A4 mutations differ among populations; p.T416P and c.1001G $>$ A are the most predominant mutations in Caucasians [17,18], p.H723R in Japanese [19] and Koreans [20], and c.919-2A>G in Han Chinese [21]. These findings underscore the importance of collecting regional data when genetic examination for $\mathrm{SNHI}$ is performed in a specific population.

Taiwan is an island in the Pacific Ocean with a population of 23.4 million people. As an immigrant population, approximately $98 \%$ of Taiwanese are of Han Chinese ancestry, whereas the remaining $2 \%$ are of aboriginal ancestry (Austronesian) [22]. According to a recent population structure study based on a nationwide biobank, the Taiwanese Han Chinese were clustered into three groups: $5 \%$ were of northern Han Chinese ancestry, 79.9\% were of southern Han Chinese ancestry, and $14.5 \%$ belonged to a third group that is genetically distinct from neighboring Southeast Asians and Austronesian tribes but similar to other southern Han Chinese [23]. As such, the predominant majority of the Taiwanese identify themselves as Han Chinese.

To address the genetic epidemiology of $\mathrm{HHI}$ in the Taiwanese population, we have established a large SNHI cohort composed of $>5000$ families in Taiwan over the past 15 years. In addition to achieving genetic diagnoses for $\mathrm{HHI}$ in these SNHI families, our results also demonstrated certain translational applications of genetic examination for HHI in clinical practice [24,25]. In this article, we analyzed the genetic profiles in this large Taiwanese cohort and reported the clinical features associated with specific genetic mutations

\section{Materials and Methods}

\subsection{Subject Recruitment and Phenotype Characterization}

From 2005 to 2019, a total of 5314 unrelated families were enrolled in the SNHI cohort of the Department of Otolaryngology, National Taiwan University Hospital. At least one member of each family suffered from idiopathic SNHI. Patients were excluded if they (1) were more than 40 years old, (2) had conductive or mixed-type hearing impairment, (3) had a history of perinatal insults, such as prematurity or kernicterus, (4) had previous noise or ototoxic agents exposure, or (5) had no complete records of their medical history available. All participants in this study identified themselves as Han Chinese. There were 198 families with multiple affected members, including 62 families compatible with autosomal dominant inheritance, 123 families compatible with autosomal recessive inheritance, and 13 families compatible with $X$-linked inheritance. The probands of most families had non-syndromic SNHI; however, some probands revealed clinical features compatible with specific syndromes, such as 
branchio-oto-renal syndrome $(n=15)$, Waardenburg syndrome $(n=13)$, Alport syndrome $(n=9)$, and Usher syndrome $(n=10)$.

For the affected member(s) of each family, a comprehensive family history, personal medical history, physical examination, audiological results, and imaging results (when indicated) were ascertained. The audiological results were evaluated with pure tone audiograms, otoacoustic emissions, auditory brainstem response, or auditory steady-state response, depending on age or neurological status [26]. Imaging results were obtained using high-resolution computed tomography and/or magnetic resonance imaging; abnormalities of the inner ear and cochlear nerve were determined according to criteria in the literature [27-29]. Informed consent were provided by all subjects and/or their parents prior to the genetic testing, and all procedures were approved by the Research Ethics Committees of the National Taiwan University Hospital.

All subjects gave their informed consent for inclusion before they participated in the study. The study was conducted in accordance with the Declaration of Helsinki, and the protocol was approved by the Ethics Committee of 201712138RINA (Project identification code).

\subsection{Conventional Genetic Examination Using Sanger Sequencing}

Since 2005, we have standardized a genetic examination protocol for mutation screening for three common deafness genes: GJB2, SLC26A4, and MTRNR1 [30-32]. Sanger sequencing was performed on exon 2 of GJB2, all 21 exons of SLC26A4, and the mitochondrial 12S rRNA gene (MTRNR1).

\subsection{Next-Generation Sequence (SGS)-Based Genetic Examination}

Since 2013, we have started to develop diagnostic panels using next-generation sequencing (NGS) technology [33]. We have been upgrading our NGS-based diagnostic panels continuously: the initial version included 80 deafness genes, which has gradually been expanded to 213 genes in our current version. The NGS-based diagnostic panel was originally designed to address families unresolved by conventional genetic examinations, but has been commercialized and utilized as a first-tier diagnostic platform since 2018.

For NGS-based genetic examinations, sample preparation, DNA sequencing, and data analyses were performed as previously described [33-36]. Briefly, paired-end reads were aligned, sorted, and converted by BWA-MEM and Picard. Variants, including single nucleotide substitution and small deletions/insertions, were called by GATK HaplotypeCaller. ANNOVAR was used to annotate pathogenicity prediction by PolyPhen-2 (HumVar), SIFT, LRT, MutationTaster, MutationAssessor, FATHMM, and MetaLR. Allele frequencies in the 1000 Genomes Project (http://www.1000genomes. org/), the Exome Aggregation Consortium projects (ExAC) (http://exac.broadinstitute.org/), and the Genome Aggregation Database (gnomAD) (http://gnomad.broadinstitute.org/) were also annotated by ANNOVAR.

After variant annotation, we identified previously reported variants through the Deafness Variation Database (http://deafnessvariationdatabase.org/, last accessed 9 January, 2019) and ClinVar (https://www.ncbi.nlm.nih.gov/clinvar/, last accessed 9 June, 2019). All variants, including reported and unreported variants, were categorized according to the American College of Medical Genetics and Genomics (ACMG) guidelines. Sanger sequencing was performed to confirm the identified variants and examine co-segregation of the genotype and SNHI phenotype among the family members. Variants fitting the criteria of "pathogenic" or "likely pathogenic" were reported as disease-causing. Genetic diagnosis was considered confirmed when either one disease-causing variant was detected in dominant genes or bi-allelic disease-causing variants were detected in recessive genes. 


\section{Results}

\subsection{Diagnostic Yields of the Genetic Examinations}

Among the probands of 5314 families, 5184 were subjected to conventional genetic examination of the three common deafness genes (Figure 1). Confirmative genetic diagnosis was achieved in 1291 (24.9\%) of the 5184 probands, including 895 (17.3\%) with bi-allelic GJB2 mutations, 294 (5.7\%) with bi-allelic SLC26A4 mutations, and 102 (2.0\%) with the m.1555A>G mutation. Of the 3893 probands without confirmed diagnosis, 280 underwent targeted NGS examination as the second phase screening, and $86(30.7 \%)$ of them were confirmed to have disease-causing variants in other deafness genes, as seen in Table 1.

From 2018 to 2019, the probands of 130 SNHI families directly received targeted NGS examination instead of conventional genetic examination. Definite genetic diagnosis was achieved in 69 (53.1\%) of the 130 probands, as seen in Table 1. Common genetic causes included mutations in GJB2 ( $n=42$, $32.3 \%)$, MYO15A $(n=8,6.2 \%)$, SLC26A4 $(n=5,3.8 \%)$, and OTOF $(n=4,3.1 \%)$. 
Table 1. Deafness genes detected with targeted NGS examination in Taiwanese patients.

\begin{tabular}{|c|c|c|c|c|}
\hline Gene & $\begin{array}{l}\text { No. of } \\
\text { Patients }\end{array}$ & $\begin{array}{c}\text { Inheritance } \\
\text { Pattern }\end{array}$ & $\begin{array}{c}\text { Associated Syndrome in } \\
\text { Ref }\end{array}$ & $\begin{array}{c}\text { Associated Syndrome in } \\
\text { our Patients }\end{array}$ \\
\hline \multicolumn{5}{|c|}{86 probands with confirmed diagnosis in the second-phase targeted NGS screening } \\
\hline OTOF & 22 & AR & - & - \\
\hline MYO15A & 18 & AR & - & - \\
\hline KCNQ4 & 5 & $\mathrm{AD}$ & - & - \\
\hline POU3F4 & 4 & X-linked & - & - \\
\hline MYO7A & 5 & $\mathrm{AD} / \mathrm{AR}$ & Usher syndrome $1 \mathrm{~B}$ & Non-syndromic \\
\hline EYA1 & 7 & $\mathrm{AD}$ & $\begin{array}{l}\text { Branchio-oto-renal } \\
\text { syndrome }\end{array}$ & $\begin{array}{l}\text { Branchio-oto-renal } \\
\text { syndrome }\end{array}$ \\
\hline TECTA & 3 & $\mathrm{AD} / \mathrm{AR}$ & - & - \\
\hline MITF & 2 & $\mathrm{AD}$ & Waardenburg syndrome & Waardenburg syndromic \\
\hline POU4F3 & 2 & $\mathrm{AD}$ & - & - \\
\hline PJVK & 1 & $\mathrm{AR}$ & - & - \\
\hline COL $4 A 5$ & 2 & X-linked & Alport syndrome & Alport syndrome \\
\hline WFS1 & 2 & $\mathrm{AD} / \mathrm{AR}$ & Wolfram syndrome & Non-syndromic \\
\hline GATA3 & 2 & $\mathrm{AD}$ & HDR syndrome & Non-syndromic \\
\hline SIX5 & 1 & $\mathrm{AD}$ & $\begin{array}{l}\text { Branchio-oto-renal } \\
\text { syndrome }\end{array}$ & $\begin{array}{l}\text { Branchio-oto-renal } \\
\text { syndrome }\end{array}$ \\
\hline TMPRSS3 & 2 & AR & - & - \\
\hline PAX3 & 1 & $\mathrm{AD}$ & Waardenburg syndrome & Waardenburg syndrome \\
\hline USH $2 A$ & 2 & AR & Usher syndrome 2A & - \\
\hline MYO6 & 2 & $\mathrm{AD} / \mathrm{AR}$ & - & - \\
\hline OTOG & 1 & AR & - & - \\
\hline ATP6V1B2 & 1 & $\mathrm{AD}$ & $\begin{array}{l}\text { Congenital deafness with } \\
\text { onychodystrophy }\end{array}$ & $\begin{array}{c}\text { Congenital deafness with } \\
\text { onychodystrophy }\end{array}$ \\
\hline SOX10 & 1 & $\mathrm{AD}$ & Waardenburg syndrome & Waardenburg syndrome \\
\hline \multicolumn{5}{|c|}{69 probands with confirmed diagnosis in directly targeted NGS screening } \\
\hline GJB2 & 42 & AR & - & - \\
\hline MYO15A & 8 & AR & - & - \\
\hline SLC26A4 & 5 & AR & Pendred syndrome & Non-syndromic \\
\hline OTOF & 4 & AR & - & \\
\hline EYA1 & 2 & $\mathrm{AD}$ & $\begin{array}{l}\text { Branchio-oto-renal } \\
\text { syndrome }\end{array}$ & $\begin{array}{l}\text { Branchio-oto-renal } \\
\text { syndrome }\end{array}$ \\
\hline KCNQ4 & 1 & $\mathrm{AD}$ & - & - \\
\hline POU3F4 & 1 & X-linked & - & - \\
\hline MYO7A & 1 & $\mathrm{AD} / \mathrm{AR}$ & Usher syndrome, type 1B & Non syndromic \\
\hline MITF & 1 & $\mathrm{AD}$ & Waardenburg syndrome & Waardenburg syndrome \\
\hline PAX3 & 1 & $\mathrm{AD}$ & Waardenburg syndrome & Waardenburg syndrome \\
\hline$E D N R B$ & 1 & $\mathrm{AD}$ & - & - \\
\hline EPS8L2 & 1 & AR & - & - \\
\hline PTPRQ & 1 & $\mathrm{AD} / \mathrm{AR}$ & - & - \\
\hline
\end{tabular}




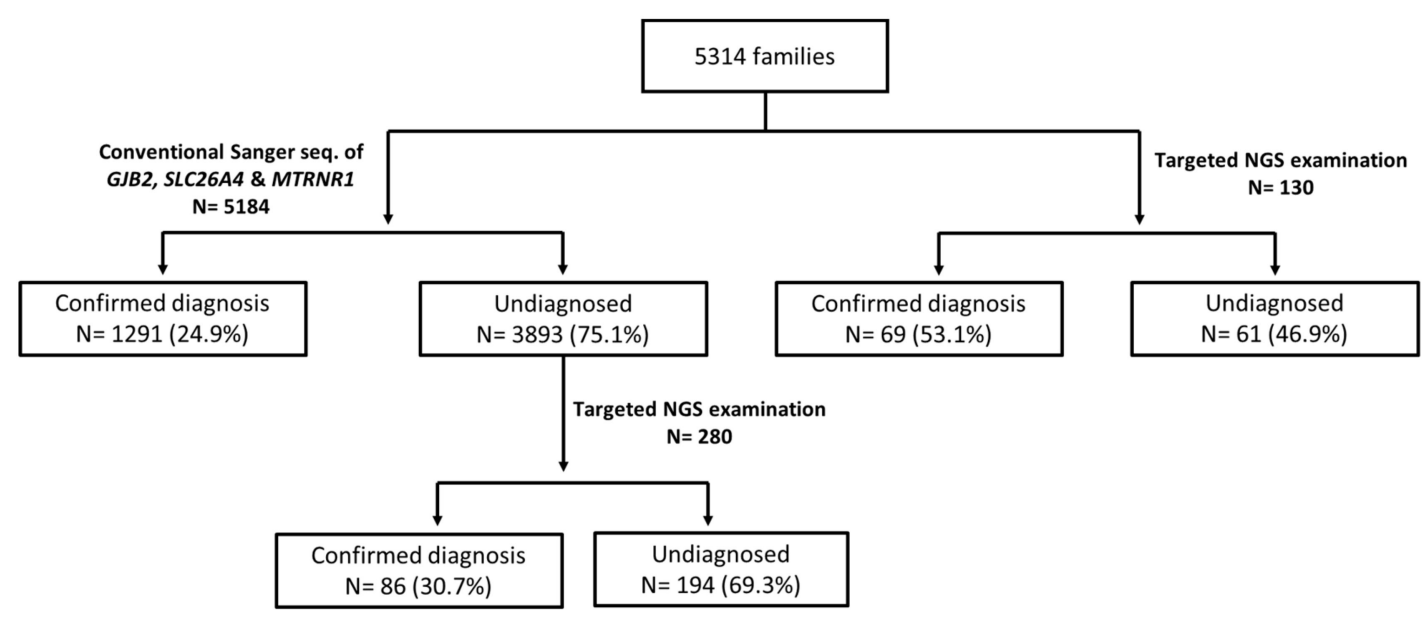

Figure 1. Summary of the genetic results in the 5314 Taiwanese families included in this study. The probands of 5184 families received conventional Sanger sequencing of three common deafness genes (i.e., GJB2, SLC26A4, and MTRNR1), and 1291 (24.9\%) of them had confirmed diagnosis. Among the remaining 3893 undiagnosed probands, 280 of them further received a second-phase targeted NGS examination, and $86(30.7 \%)$ had confirmed diagnosis. On the other hand, 130 families directly received targeted NGS examination without screening of common deafness gene a priori. Of them, 69 (53.1\%) had confirmed diagnosis. NGS, next-generation sequencing.

The allele frequencies of mutations in deafness genes in the Taiwanese families are shown in Figure 2. The most prevalent pathogenic variants included GJB2 mutations (22.92\%), SLC26A4 mutations $(6.03 \%)$, OTOF mutations (4.62\%), MYO15A mutations (2.98\%), and the m.1555A $>$ G mutation (1.95\%).

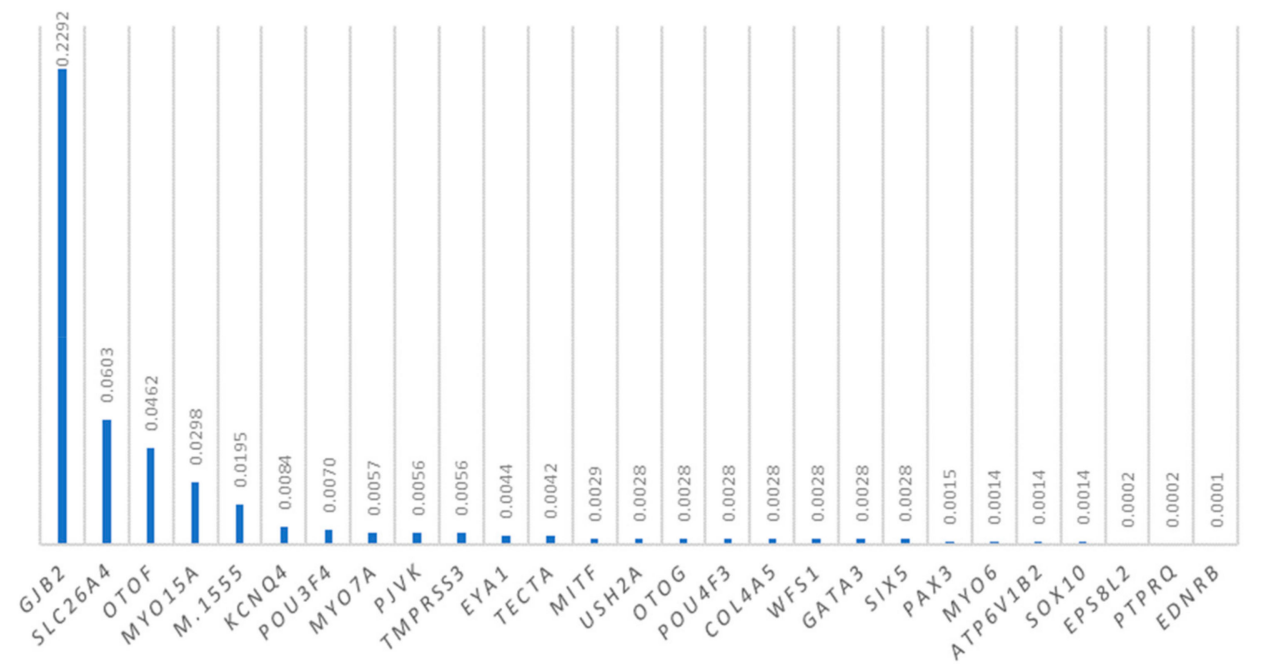

Figure 2. Allele frequencies of deafness-associated genetic mutations in the Taiwanese families.

\subsection{GJB2 Mutations}

In our cohort, the probands of 935 families were diagnosed to have bi-allelic recessive GJB2 mutations, and two probands were diagnosed to have mono-allelic dominant GJB2 mutations. The most common GJB2 mutations in the Taiwanese SNHI probands included p.V37I (85.9\%), c.235delC (11.5\%), and c.299_300delAT (1.7\%). The GJB2 mutations identified in the 937 Taiwanese families are shown in Table 2. 
Table 2. GJB2 mutations detected in the Taiwanese patients.

\begin{tabular}{cccc}
\hline Nucleotide Change & Amino Acid Change & Allele Count (ratio\%) & $\begin{array}{c}\text { Popmax AF (population) } \\
\text { from gnomAD }\end{array}$ \\
\hline Recessive mutations & & & \\
c.109G $>$ A & p.V37I & $1608(85.9)$ & 0.08345 (EAS) \\
c.235delC & p.L79Cfs *3 & $215(11.5)$ & 0.006515 (EAS) \\
c.299_300delAT & p.H100Rfs ${ }^{*} 14$ & $31(1.7)$ & 0.0009023 (EAS) \\
c.427C $>$ T & p.R143W & $6(0.3)$ & 0.0007227 (AFR) \\
c.508_511dupAACG & p.A171Qfs ${ }^{*} 40$ & $5(0.3)$ & 0.0003008 (EAS) \\
c.176_191del & p.G59Afs ${ }^{*} 18$ & $2(0.1)$ & 0.0001631 (EAS) \\
c.95G $>$ A & p.R32H & $1(0.1)$ & 0.000008859 (EUP-NF) \\
c.107T $>$ C & p.L36P & $1(0.1)$ & 0.0001002 (EAS) \\
c.571T $>$ C & p.F191L & $1(0.1)$ & 0.001854 (EAS) \\
Dominant mutations & p.A88V & $1(0.1)$ & no data \\
c.263C $>$ T & p.R143Q & $1(0.1)$ & no data \\
c.428G $>$ A & & $1872(100.0)$ & \\
\hline Total & &
\end{tabular}

NCBI Reference Sequence: NM_004004.6/NP_003995.2. *: termination; del: deletion; dup: duplication. EAS: East Asian; AFR: African; EUP-NF: European-non-Finnish.

Different GJB2 genotypes were associated with different hearing severity and audiogram shapes. Patients who were homozygous or compound heterozygous for p.V37I showed milder SNHI than those with other GJB2 mutations. Nonetheless, progressive SNHI could occur in patients with different GJB2 genotypes with an average progression rate of $\sim 0.55 \mathrm{dBHL}$ per year. Notably, the progression rate did not differ significantly among patients with different GJB2 genotypes [37].

\subsection{SLC26A4 Mutations}

Clinically, SLC26A4 mutations contribute to non-syndromic enlarged vestibular aqueduct (DFNB4, MIM 600791) and Pendred syndrome (PS, MIM 274600). To date, we have performed comprehensive genetic screening for SLC26A4 in the probands of 361 families with DFNB4 or PS. In total, SLC26A4 mutations were detected in 346 probands, including $300(83.1 \%)$ with bi-allelic and $46(12.7 \%)$ with mono-allelic SLC26A4 mutations. The SLC26A4 mutations identified in the 346 Taiwanese families are shown in Table 3. We did not observe differences in clinical features, including radiological findings, the presence of goiters, and audiological results, in our patients with different SLC26A4 genotypes $[31,38]$.

\subsection{OTOF Mutations}

To date, we have performed comprehensive genetic examination in the probands of 54 families with non-acquired auditory neuropathy. The OTOF mutations identified in these families are shown in Table 4. A total of 22 probands were diagnosed with bi-allelic OTOF mutations, including eight homozygous for p.E1700Q and 14 compound heterozygous for p.E1700Q and another variant. Eight probands were found to have mono-allelic OTOF mutations. It is unclear whether auditory neuropathy in these mono-allelic probands was caused by the presence of another hidden OTOF mutation or by other etiologies. 
Table 3. SLC26A4 mutations detected in 346 Taiwanese families with DFNB4/PS.

\begin{tabular}{|c|c|c|c|}
\hline Nucleotide Change & Amino Acid Change & Allele Count (ratio\%) & $\begin{array}{c}\text { Popmax AF (population) } \\
\text { from gnomAD }\end{array}$ \\
\hline \multicolumn{4}{|c|}{ Bi-allelic SLC26A4 mutations } \\
\hline c. $919-2 \mathrm{~A}>\mathrm{G}$ & NA & $473(73.2)$ & 0.005064 (EAS) \\
\hline c. $2168 \mathrm{~A}>\mathrm{G}$ & p.H723R & $39(6.0)$ & 0.001604 (EAS) \\
\hline c. $1229 \mathrm{C}>\mathrm{T}$ & p.T410M & $17(2.6)$ & 0.0005879 (SA) \\
\hline c. $1160 \mathrm{C}>\mathrm{T}$ & p.A387V & $6(0.9)$ & 0.00005438 (EAS) \\
\hline c. $754 \mathrm{~T}>\mathrm{C}$ & p.S252P & $5(0.8)$ & 0.00005437 (EAS) \\
\hline c. $1115 \mathrm{C}>\mathrm{T}$ & p.A372V & $5(0.8)$ & No data \\
\hline c.916dupG & p.V306Gfs *24 & $5(0.8)$ & 0.0001631 (EAS) \\
\hline c. $164+1 G>C$ & NA & $4(0.6)$ & No data \\
\hline c. $706 \mathrm{C}>\mathrm{G}$ & p.L236V & $3(0.5)$ & $0.0002602(\mathrm{LAT})$ \\
\hline c. $1343 \mathrm{C}>\mathrm{T}$ & p.S448L & $3(0.5)$ & 0.0001088 (EAS) \\
\hline c. $439 \mathrm{~A}>\mathrm{G}$ & p.M147V & $2(0.3)$ & 0.0001087 (EAS) \\
\hline c. $1079 \mathrm{C}>\mathrm{T}$ & p.A360V & $2(0.3)$ & 0.000641 (EAS) \\
\hline c. $1173 \mathrm{C}>\mathrm{A}$ & p.S391R & $2(0.3)$ & No data \\
\hline c. $1318 \mathrm{~A}>\mathrm{T}$ & p.K $440 *$ & $2(0.3)$ & No data \\
\hline c. $1489 \mathrm{G}>\mathrm{C}$ & p.G497R & $2(0.3)$ & 0.00005013 (EAS) \\
\hline c. $2086 \mathrm{C}>\mathrm{T}$ & p.Q696* & $2(0.3)$ & 0.0001695 (EAS) \\
\hline c. $2 \mathrm{~T}>\mathrm{G}$ & p.M1R & $1(0.2)$ & 0.00007383 (EAS) \\
\hline c. $230 \mathrm{~A}>\mathrm{T}$ & p.K77I & $1(0.2)$ & No data \\
\hline c. $235 \mathrm{C}>\mathrm{T}$ & p.R79 * & $1(0.2)$ & No data \\
\hline c. $241 \mathrm{~A}>\mathrm{G}$ & p.K81E & $1(0.2)$ & No data \\
\hline c.387delC & p.F130Lfs *15 & $1(0.2)$ & No data \\
\hline c. $416-1 G>A$ & NA & $1(0.2)$ & 0.00005437 (EAS) \\
\hline c. $697 \mathrm{G}>\mathrm{C}$ & p.V233L & $1(0.2)$ & $0.001353($ EAS) \\
\hline c. $918+2 \mathrm{~T}>\mathrm{C}$ & NA & $1(0.2)$ & $0.0001387(\mathrm{OTH})$ \\
\hline c. $1001+1 G>A$ & NA & $1(0.2)$ & 0.0003977 (EUP-NF) \\
\hline c. $1105 \mathrm{~A}>\mathrm{T}$ & p.K369* & $1(0.2)$ & No data \\
\hline c. $1174 \mathrm{~A}>\mathrm{T}$ & p.N392Y & $1(0.2)$ & 0.00005438 (EAS) \\
\hline c. $1226 \mathrm{G}>\mathrm{A}$ & p.R409H & $1(0.2)$ & 0.0001977 (LAT) \\
\hline c. $1489 \mathrm{G}>\mathrm{A}$ & p.G497S & $1(0.2)$ & 0.00005013 (EAS) \\
\hline c.1520delT & p.L507* & $1(0.2)$ & No data \\
\hline c. $1544+6 \mathrm{~T}>\mathrm{C}$ & NA & $1(0.2)$ & No data \\
\hline c. $1614+1 G>A$ & NA & $1(0.2)$ & 0.00003525 (EUP-NF) \\
\hline c. $1615-2 A>G$ & NA & $1(0.2)$ & 0.000008827 (EUP-NF) \\
\hline c. $1658 \mathrm{C}>\mathrm{A}$ & p.P553H & $1(0.2)$ & $0.00005438(\mathrm{EAS})$ \\
\hline c.1693_1694insA & p.C565* & $1(0.2)$ & No data \\
\hline c. $170 \overline{7}+1 \mathrm{G}>\mathrm{A}$ & NA & $1(0.2)$ & No data \\
\hline c. $1786 \mathrm{C}>\mathrm{T}$ & p.Q596* & $1(0.2)$ & No data \\
\hline c. $1829 \mathrm{C}>\mathrm{A}$ & p.S610* & $1(0.2)$ & No data \\
\hline c. $1975 G>C$ & p.V659L & $1(0.2)$ & 0.0002006 (EAS) \\
\hline c. $2086 \mathrm{C}>\mathrm{T}$ & p.Q696T * & $1(0.2)$ & 0.0001695 (EAS) \\
\hline c. $2089 \mathrm{G}>\mathrm{C}$ & p.D697H & $1(0.2)$ & No data \\
\hline c. $2107 \mathrm{C}>\mathrm{G}$ & p.L703V & $1(0.2)$ & No data \\
\hline c. $2162 \mathrm{C}>\mathrm{T}$ & p.T721M & $1(0.2)$ & 0.0002895 (LAT) \\
\hline g.-1066C_6602Adel & NA & $1(0.2)$ & No data \\
\hline \multicolumn{4}{|c|}{ Mono-allelic SLC26A4 mutations } \\
\hline c. $919-2 \mathrm{~A}>\mathrm{G}$ & NA & $44(6.8)$ & 0.005064 (EAS) \\
\hline c.916_917insG & p.V306Gfs *24 & $1(0.2)$ & 0.0001631 (EAS) \\
\hline $\begin{array}{c}\text { c.974_977 } \\
\text { delCTGGinsTTAAATTA }\end{array}$ & p.A325Vfs *6 & $1(0.2)$ & No data \\
\hline Total & & $646(100.0)$ & \\
\hline
\end{tabular}

NCBI Reference Sequence: NM_000441.1/NP_000432.1. del: deletion; ins: insertion; dup: duplication; *: termination; fs: frameshift. EAS: East Asian; SA: South Asian; LAT: Latino; EUP-NF: European-non-Finnish; OTH: other. 
Table 4. OTOF mutations detected in 30 Taiwanese families with auditory neuropathy.

\begin{tabular}{|c|c|c|c|}
\hline Nucleotide Change & Amino Acid Change & Allele Count (ratio\%) & $\begin{array}{c}\text { Popmax AF (population) } \\
\text { from gnomAD }\end{array}$ \\
\hline \multicolumn{4}{|c|}{ Bi-allelic OTOF mutations } \\
\hline c. $5098 \mathrm{G}>\mathrm{C}$ & p.E1700Q & $30(57.7)$ & 0.006774 (EAS) \\
\hline c. $2521 \mathrm{G}>\mathrm{A}$ & p.E841K & $3(5.8)$ & 0.0002202 (EAS) \\
\hline c. $1498 \mathrm{C}>\mathrm{T}$ & p.R500* & $1(1.9)$ & $0.0001387(\mathrm{OTH})$ \\
\hline c. $2279 \mathrm{~T}>\mathrm{C}$ & p.L760P & $1(1.9)$ & no data \\
\hline c.3704_3719del & p.D1235Afs *30 & $1(1.9)$ & no data \\
\hline c. $3894+5 \mathrm{G}>\mathrm{C}$ & NA & $1(1.9)$ & no data \\
\hline c. $4023+1 G>A$ & NA & $1(1.9)$ & 0.01178 (EAS) \\
\hline c. $4030 \mathrm{C}>\mathrm{T}$ & p.R1344* & $1(1.9)$ & no data \\
\hline c. $4961-1 \mathrm{G}>\mathrm{A}$ & NA & $1(1.9)$ & no data \\
\hline c. $5197 \mathrm{G}>\mathrm{A}$ & p.E1733K & $1(1.9)$ & 0.00005439 (EAS) \\
\hline c. $5203 \mathrm{C}>\mathrm{T}$ & p.R1735W & $1(1.9)$ & 0.000008792 (EUP-NF) \\
\hline c. $5335 \mathrm{C}>\mathrm{T}$ & p.H1779Y & $1(1.9)$ & no data \\
\hline c. $.5566 \mathrm{C}>\mathrm{T}$ & p.R1856W & $1(1.9)$ & 0.0001003 (EAS) \\
\hline \multicolumn{4}{|c|}{ Mono-allelic OTOF mutations } \\
\hline c. $5098 \mathrm{G}>\mathrm{C}$ & p.E1700Q & $6(11.5)$ & 0.006774 (EAS) \\
\hline c. $4023+1 G>A$ & NA & $1(1.9)$ & 0.01178 (EAS) \\
\hline c. $4227+5 G>C$ & NA & $1(1.9)$ & 0.001414 (EAS) \\
\hline Total & & $52(100.0)$ & \\
\hline
\end{tabular}

NCBI Reference Sequence: NM_194248.2/NP_919224.1. *: termination; fs: frameshift; del: deletion; EAS: East Asian; EUP-NF: European-non-Finnish; OTH: other.

In our cohort, all patients with bi-allelic OTOF mutations presented phenotypes compatible with auditory neuropathy of autosomal recessive inheritance. The hearing thresholds on behavioral audiometries ranged from moderate to profound SNHI, with two-thirds of them showing moderate SNHI and one-third showing severe-to-profound SNHI at initial presentation. Most of the patients $(75 \%)$ failed newborn hearing screening at birth. In the twelve patients with longitudinal hearing data available for analyses, seven (58.3\%) showed progressive hearing loss, four (33.3\%) showed stable hearing loss, and one ( $8.3 \%)$ demonstrated fluctuation in the hearing levels.

\subsection{MYO15A Mutations}

Recessive MYO15A mutations are the fourth most common genetic cause of SNHI in Taiwanese patients. In our cohort, a total of 22 families (27 patients) were diagnosed to have bi-allelic pathogenic or likely pathogenic variants in MYO15A. The MYO15A mutations identified in these Taiwanese patients are shown in Table 5. The most prevalent variants were c.3524dupA (20.5\%, 9/44), c.8182C $>$ G $(9.1 \%, 4 / 44)$, and c.3757-32_3757-1del $(6.8 \%, 3 / 44)$. Several variants are unique to the Taiwanese patients and have never been identified in other populations, including c.3757-32_3757-1del, c.4101C >A, c.4457G > T, c.4760T>C, c.4761_4762insGTTTCTAT, c.5443C >A, c.6956+1G>A, c.8182C >G, c. $8602-1 \mathrm{G}>\mathrm{C}$, and c. $9408 \mathrm{G}>\mathrm{C}$.

In our cohort, more than half $(77.8 \%, 21 / 27)$ of the patients with bi-allelic MYO15A mutations presented with severe to profound SNHI. Patients with mutations in the N-terminal domain showed less severe $\mathrm{SNHI}$ and more low-tone residual hearing compared to those with mutations in the non-N terminal domain (unpublished data). Approximately one-third of our patients showed progression of SNHI during the follow-up period. None of them developed vestibular symptoms. 
Table 5. MYO15A mutations detected in Taiwanese patients.

\begin{tabular}{|c|c|c|c|}
\hline Nucleotide Change & Amino Acid Change & Allele Count (ratio\%) & $\begin{array}{c}\text { Popmax AF (population) } \\
\text { from gnomAD }\end{array}$ \\
\hline c.3524dupA & p.Ser1176ValfsTer14 & $9(20.5)$ & 0.002152 (EAS) \\
\hline c.10250_10252delCCT & p.Ser3417del & $8(18.2)$ & 0.0004095 (EAS) \\
\hline c. $8182 \mathrm{C}>\mathrm{G}$ & p.Arg2728Gly & $4(9.1)$ & 0.0002226 (EAS) \\
\hline c.3757-32_3757-1del & NA & $3(6.8)$ & 0.0003583 (EAS) \\
\hline c. $5964+3 \mathrm{G}>\mathrm{A}$ & NA & $2(4.5)$ & 0.0003908 (EAS) \\
\hline c. $9408 \mathrm{G}>\mathrm{C}$ & p.Trp3136Cys & $2(4.5)$ & 0.0003583 (EAS) \\
\hline c. $10111 C>\mathrm{T}$ & p.G $\ln 3371$ * & $1(2.3)$ & 0.00006473 (AFR) \\
\hline c.10258_10260delTTC & p.Phe3420del & $1(2.3)$ & 0.0001112 (EAS) \\
\hline c. $3844 \mathrm{C}>\mathrm{T}$ & p.Arg1282Trp & $1(2.3)$ & 0.0001403 (OTH) \\
\hline c. $4101 \mathrm{C}>\mathrm{A}$ & p.Asn1367Lys & $1(2.3)$ & no data \\
\hline c. $4457 \mathrm{G}>\mathrm{T}$ & p.Gly1486Val & $1(2.3)$ & no data \\
\hline c. $4642 \mathrm{G}>\mathrm{A}$ & p.Ala1548Thr & $1(2.3)$ & 0.0001239 (AFR) \\
\hline c. $4760 \mathrm{~T}>\mathrm{C}$ & p.Leu1587Pro & $1(2.3)$ & no data \\
\hline c.4761_4762insGTTTCTAT & p.Asp1588Valfs *11 & $1(2.3)$ & no data \\
\hline c.5421_5437del & p.Glu1808Glyfs *41 & $1(2.3)$ & no data \\
\hline c. $5443 \mathrm{C}>\mathrm{A}$ & p.Gln1815Lys & $1(2.3)$ & no data \\
\hline c. $5977 \mathrm{C}>\mathrm{T}$ & p.Arg1993Trp & $1(2.3)$ & 0.0001712 (EAS) \\
\hline c. $6281 \mathrm{G}>\mathrm{A}$ & p.Arg2094His & $1(2.3)$ & 0.000008965 (EUP-NF) \\
\hline c. $6956+1 G>A$ & NA & $1(2.3)$ & 0.00006504 (EAS) \\
\hline c.7708_7709insCA & p.Gln2571Hisfs * 35 & $1(2.3)$ & 0.000641 (EAS) \\
\hline c.7986dupG & p.Glu2663Glyfs * 4 & $1(2.3)$ & no data \\
\hline c. $8602-1 G>C$ & NA & $1(2.3)$ & no data \\
\hline Total & & $52(100.0)$ & \\
\hline
\end{tabular}

NCBI Reference Sequence: NM_016239.4/NP_057323.3. dup: duplication; del: deletion; ins: insertion; *: termination; fs: frameshift. EAS: East Asian; AFR: African; EUP-NF: European-non-Finnish; OTH: other.

\subsection{MTRNR1 Mutations}

The m.1555A $>\mathrm{G}$ mutation in the MTRNR1 gene accounted for $\sim 2 \%$ of Taiwanese families ( $\sim 10 \%$ of multiplex families) with idiopathic SNHI [39]. The m.1494C $>$ T mutation that has been reported as pathogenic in other populations $[40,41]$ was not detected in our cohort. In Taiwanese patients, m.1555A $>\mathrm{G}$ was identified in a variety of mtDNA backgrounds (i.e., the mitochondrial haplogroups), suggesting that this mutation arose from multiple origins in the Taiwanese population [39]. Interestingly, families with various haplogroups demonstrated different penetrance of SNHI, indicating that the mtDNA background might exert effects on the disease expression [39].

\subsection{Mutations in Other Deafness Genes}

In our cohort, pathogenic variants were also detected in a number of other deafness genes, including KCNQ4, POU3F4, MYOTA, TMPRSS3, EYA1, TECTA, MITF, POU4F3, PJVK, COL4A5, WFS1, GATA3, SIX5, USH2A, OTOG, PAX3, MYO6, ATP6V1B2, SOX10, PTPRQ, EPS8L2, and EDNRB, as shown in Table 6. Some variants are unique to Taiwanese patients and have never been identified in other populations.

Of these, mutations in 15 genes, including KCNQ4, MYO7A, EYA1, TECTA, MITF, POU4F3, WFS1, GATA3, SIX5, PAX3, MYO6, ATP6V1B2, SOX10, EDNRB, and PTPRQ are associated with autosomal dominant inheritance; mutations in 12 genes are associated with syndromic hearing loss, including Waardenberg syndrome (MITF, PAX3, and SOX10), BOR syndrome (SIX5 and EYA1), hypoparathyroidism, sensorineural deafness, and renal dysplasia (HDR) syndrome (GATA3), Wolfram syndrome (WFS1), Usher syndrome (USH2A and MYO7A), Pendred syndrome (SLC26A4), Alport syndrome (COL4A5), congenital deafness with onychodystrophy (ATP6V1B2), as shown in Table 1. 
Table 6. Mutations of other deafness genes detected in Taiwanese patients.

\begin{tabular}{|c|c|c|c|c|}
\hline Nucleotide Change & Amino Acid Change & Allele Count & $\begin{array}{l}\text { Popmax AF (population) } \\
\text { from gnomAD }\end{array}$ & References \\
\hline \multicolumn{5}{|l|}{ KCNQ4 } \\
\hline c. $546 C>\mathrm{G}$ & p.F182L & 3 & 0.004576 (EAS) & Our cohort; Ref [42] \\
\hline c. $2014 \mathrm{G}>\mathrm{A}$ & p.V672M & 2 & No data & Our cohort \\
\hline c. $2039 \mathrm{C}>\mathrm{T}$ & p.S680F & 1 & 0.0002006 (EAS) & Our cohort; Ref [33] \\
\hline \multicolumn{5}{|l|}{ MYOTA } \\
\hline c. $689 \mathrm{C}>\mathrm{T}$ & p.A230V & 1 & No data & Our cohort \\
\hline c. $1142 \mathrm{C}>\mathrm{T}$ & p.T381M & 1 & 0.0007619 (EAS) & $\begin{array}{c}\text { Our cohort; Ref } \\
{[33,43]}\end{array}$ \\
\hline c. $2557 \mathrm{C}>\mathrm{T}$ & p.R853C & 1 & No data & Our cohort \\
\hline c. $6335 \mathrm{C}>\mathrm{G}$ & p.S2112* & 1 & No data & Our cohort \\
\hline c. $6470 \mathrm{~T}>\mathrm{C}$ & p.I2157T & 1 & 0.00005563 (EAS) & Our cohort \\
\hline c. $592+1 \mathrm{G}>\mathrm{A}$ & NA & 1 & 0.00005563 (EAS) & Our cohort \\
\hline \multicolumn{5}{|l|}{ POU3F4 } \\
\hline c.346dupG & p.A116Gfs *77 & 2 & No data & Our cohort \\
\hline c.919_921delGAG & p.E307del & 1 & No data & Our cohort \\
\hline c. $950 \mathrm{~T}>\mathrm{A}$ & p.L317* & 1 & No data & Our cohort \\
\hline c. $1084 \mathrm{~T}>\mathrm{C}$ & p. $* 362 \operatorname{Rext} * 113$ & 1 & No data & Our cohort \\
\hline \multicolumn{5}{|l|}{ EYA1 } \\
\hline c. $1081 \mathrm{C}>\mathrm{T}$ & p.R361* & 2 & No data & Our cohort; Ref [36] \\
\hline c.1540_1542delCTG & p.L514del & 2 & No data & Our cohort \\
\hline c. $385 \mathrm{C}>\mathrm{T}$ & p.Q129* & 1 & No data & Our cohort \\
\hline c. $403 \mathrm{G}>\mathrm{A}$ & p.G135S & 1 & 0.0007082 (EAS) & Our cohort \\
\hline c. $466 \mathrm{C}>\mathrm{T}$ & p.Q156* & 1 & No data & Our cohort; Ref [44] \\
\hline c. $1360+5 \mathrm{G}>\mathrm{A}$ & NA & 1 & No data & Our cohort; Ref [36] \\
\hline c. $1735 \mathrm{delG}$ & p.D579Tfs *60 & 1 & No data & Our cohort; Ref [44] \\
\hline \multicolumn{5}{|l|}{ TECTA } \\
\hline c. $5372 C>G$ & p.P1791R & 1 & 0.001035 (EAS) & Our cohort \\
\hline c. $5471 \mathrm{G}>\mathrm{A}$ & p.G1824D & 1 & 0.0005513 (EAS) & Our cohort \\
\hline c. $6062 \mathrm{G}>\mathrm{A}$ & p.R2021H & 1 & 0.000008791 (EUP-NF) & Our cohort \\
\hline \multicolumn{5}{|l|}{ POU4F3 } \\
\hline c. $491 \mathrm{C}>\mathrm{G}$ & p.P164R & 1 & 0.0004023 (EAS) & Our cohort \\
\hline c. $982 \mathrm{~A}>\mathrm{G}$ & p.K328E & 1 & No data & Our cohort; Ref [35] \\
\hline \multicolumn{5}{|l|}{ MITF } \\
\hline c.862dupA & p.I288Nfs *9 & 1 & No data & Our cohort \\
\hline c. $1078 \mathrm{C}>\mathrm{T}$ & p.R360* & 1 & No data & Our cohort \\
\hline c. $938-1 \mathrm{G}>\mathrm{A}$ & NA & 1 & No data & Our cohort \\
\hline \multicolumn{5}{|l|}{ PJVK } \\
\hline c. $406 \mathrm{C}>\mathrm{T}$ & p.R136* & 1 & 0.00008316 (AFR) & Our cohort \\
\hline c. $593 \mathrm{C}>\mathrm{A}$ & p.A198D & 1 & 0.0005119 (EAS) & Our cohort \\
\hline \multicolumn{5}{|l|}{ COL4A5 } \\
\hline c. $367 \mathrm{delG}$ & p.G123Dfs *32 & 1 & No data & Our cohort \\
\hline c. $796 \mathrm{C}>\mathrm{T}$ & p.R266 * & 1 & No data & Our cohort \\
\hline \multicolumn{5}{|l|}{ WFS1 } \\
\hline c. $2051 C>T$ & p.A684V & 2 & No data & Our cohort \\
\hline \multicolumn{5}{|l|}{ SIX 5} \\
\hline c.1872dupC & p.A625Rfs *15 & 1 & No data & Our cohort \\
\hline \multicolumn{5}{|l|}{ GATA3 } \\
\hline c.149delT & p.F51Lfs *144 & 1 & No data & Our cohort; Ref [34] \\
\hline c. $477 \mathrm{delG}$ & p.D160Tfs *35 & 1 & No data & Our cohort \\
\hline \multicolumn{5}{|l|}{ TMPRSS3 } \\
\hline c. $916 \mathrm{G}>\mathrm{A}$ & p.A306T & 2 & 0.0006014 (EAS) & Our cohort \\
\hline c. 432 delA & p.Q144Hfs *8 & 1 & 0.0005012 (EAS) & Our cohort \\
\hline c. $743 \mathrm{C}>\mathrm{T}$ & p.T248M & 1 & 0.0004894 (EAS) & Our cohort \\
\hline \multicolumn{5}{|l|}{ PAX3 } \\
\hline c. $52 \mathrm{C}>\mathrm{T}$ & p.Q18* & 1 & No data & Our cohort \\
\hline c. $587-2 A>G$ & NA & 1 & 0.000109 (EAS) & Our cohort \\
\hline \multicolumn{5}{|l|}{ USH $2 A$} \\
\hline c. $1614 \mathrm{C}>\mathrm{A}$ & p.C538* & 1 & No data & Our cohort \\
\hline c. $4576 \mathrm{G}>\mathrm{A}$ & p.G1526R & 1 & 0.0002521 (EAS) & Our cohort \\
\hline c.7045dupT & p.W2349Lfs *8 & 1 & No data & Our cohort \\
\hline c. $8559-2 A>G$ & NA & 1 & 0.0004353 (EAS) & Our cohort \\
\hline
\end{tabular}


Table 6. Cont.

\begin{tabular}{|c|c|c|c|c|}
\hline Nucleotide Change & Amino Acid Change & Allele Count & $\begin{array}{l}\text { Popmax AF (population) } \\
\text { from gnomAD }\end{array}$ & References \\
\hline \multicolumn{5}{|l|}{ MYO6 } \\
\hline c.3736delC & p.P1246Qfs *39 & 1 & No data & Our cohort \\
\hline c. $1675-2 \mathrm{~A}>\mathrm{C}$ & NA & 1 & No data & Our cohort \\
\hline \multicolumn{5}{|l|}{ OTOG } \\
\hline c. $3582 \mathrm{C}>\mathrm{A}$ & p.Y1194* & 1 & 0.0009720 (EAS) & Our cohort \\
\hline c. $4023 \_4045 \mathrm{del}$ & p.Q1342Pfs *104 & 1 & No data & Our cohort \\
\hline \multicolumn{5}{|l|}{$A T P 6 V 1 B 2$} \\
\hline $\begin{array}{l}\text { c. } 1516 \mathrm{C}>\mathrm{T} \\
\text { SOX10 }\end{array}$ & \multicolumn{3}{|c|}{ SOX10 } & Our cohort \\
\hline $\begin{array}{l}\text { c.314_315del } \\
P T P R Q\end{array}$ & p.K105Tfs *28 & 1 & No data & Our cohort \\
\hline c. $6087-3 T>G$ & NA & 3 & 0.002970 (EAS) & Our cohort \\
\hline c.3181delC & p.L1061Ffs *11 & 1 & No data & Our cohort \\
\hline \multicolumn{5}{|l|}{ EPS8L2 } \\
\hline $\begin{array}{l}\text { c. } 1304 \mathrm{G}>\mathrm{A} \\
\text { EDNRB }\end{array}$ & p.W435* & 2 & 0.0001784 (EAS) & Our cohort \\
\hline c. $754-2 A>G$ & NA & 1 & No data & Our cohort \\
\hline
\end{tabular}

All NCBI Reference Sequences are listed in Supplementary Material Table S1. del: deletion; dup: duplication; fs: frameshift; *: termination; ext: extension. EAS: East Asian; AFR: African; EUP-NF: European-non-Finnish.

\section{Discussion}

\subsection{Comparison of Diagnostic Yields among Different Examination Strategies}

Genetic diagnosis could be confirmed in approximately $25 \%$ of the SNHI families by conventional Sanger sequencing of the three common deafness genes. By contrast, the diagnosis rate increased to approximately $50 \%$ using the targeted NGS examination strategy. The diagnostic yield of the targeted NGS genetic examination is approaching the reported ratio (i.e., one-half to two-thirds) of $\mathrm{HHI}$ in pediatric SNHI [4]. To date, several NGS-based diagnostic panels for HHI, including ours, have been designed [4,45-49]. There is solid evidence that the use of the NGS technique can significantly increase the diagnostic yield of genetic examination for $\mathrm{HHI}$ and facilitate the genetic counseling for the affected families.

\subsection{GJB2 Mutations}

GJB2 mutations are the most common genetic cause of SNHI in Taiwanese families, as in many other populations [37]. To date, more than 340 pathogenic recessive or dominant GJB2 mutations have been reported (http://deafnessvariationdatabase.org/) [38]. The prevalence of GJB2 mutations in hearing-impaired patients varied significantly across different populations, ranging from $5.6 \%$ in the sub-Saharan Africa population to $27.1 \%$ in the European population [37]. The mutation spectra also differed among different ethnic groups.

Notably, the p.V37I variant was exceedingly prevalent not only in hearing-impaired patients but also in the normal Taiwanese population. According to our population-based genetic screenings in newborns, the allele frequency of p.V37I is estimated to be $8.5 \%$ in the general Taiwanese population [50,51]. A high prevalence of the p.V37I variant has also been reported in patients recruited from south China [52] and Thailand [53].

Interestingly, our recent collaboration with Erdenechuluun et al. in Mongolia revealed a unique GJB2 mutation spectrum in Mongolian patients with SNHI [54]. Three GJB2 mutations that are prevalent in other populations, including c.235delC in East Asians [5-7], c.35delG in Caucasians [8-10], and c. $-23+1 G>A$ in Southwest and South Asians [13-16], were simultaneously detected in Mongolian patients. Haplotype analyses further confirmed founder effects for each of the three mutations, indicating that each mutation was independently derived from its ancestral origin. 
The severity of GJB2-associated SNHI varies from late-onset mild hearing loss to congenital, severe-to-profound deafness, and is highly dependent on the genotypes. Patients with two "truncating" mutation alleles (such as c.235delC) usually exhibit severe-to-profound SNHI, whereas those with at least one "non-truncating" mutation allele (such as p.V37I) are associated with a milder phenotype [55-58]. Diversity in auditory phenotypes among patients with the same GJB2 genotype was also reported $[37,55,59]$. In our recent study, we used generalized estimating equation (GEE) analyses to establish a predictive model for SNHI in patients with GJB2 mutations [37]. This predictive model for SNHI may have important clinical implications in guiding follow-up protocols and designing treatment plans in patients with GJB2 mutations.

\subsection{SLC26A4 Mutations}

Recessive SLC26A4 mutations are the second most common genetic cause of SNHI in Taiwanese patients [30]. More than 400 pathogenic variants of SLC26A4 have been reported (http://deafnessvariationdatabase.org/) [60]. The mutation spectra of SLC26A4 differ significantly among different ethnic backgrounds $[17,19,61,62]$. In Taiwanese patients, the most prevalent SLC26A4 mutation is c.919-2A $>$ G [31], followed by the p.H723R mutation, which is the most common SLC26A4 mutation in Japanese $[61,63]$ and Korean $[61,62]$ patients. Haplotype analyses confirmed the founder effect of c.919-2A $>\mathrm{G}$ in Taiwanese patients [31].

SLC26A4 mutations are distributed over all 21 exons of the gene and are mostly single nucleotide variations, yet copy number variations were occasionally reported [36,64-66]. As such, genetic examination using conventional sequencing strategies is highly laborious and may fail to detect the pathogenic variants. On the contrary, our recent study demonstrated the utility of a NGS-based panel in addressing SLC26A4-related SNHI by identifying various types of mutations with satisfactory diagnostic yields in one step [36].

Approximately $83 \%$ of the DFNB4/PS patients in our cohort were confirmed to have bi-allelic SLC26A4 mutations, which was comparable to that observed in Japanese (47-66\%) [19,67], Korean (81\%) [20], and Chinese (65-88\%) [68,69] patients, and significantly higher than that observed in Caucasian patients (16-24\%) [70,71]. Although positive genotype-phenotype correlations have been documented in some series $[67,71,72]$, no differences in clinical features were observed in our patients with different SLC26A4 genotypes [31,38]. Specifically, patients with mono-allelic SLC26A4 mutations demonstrated clinical features undistinguishable from those with bi-allelic SLC26A4 mutations, implying that there might be an undetected mutation in the no-mutation-detected SLC26A4 allele in these heterozygotes [38].

\subsection{OTOF Mutations}

OTOF mutations are another common cause of childhood SNHI, and the leading genetic cause of auditory neuropathy in Taiwanese patients, as in other populations [73,74]. p.E1700Q is the predominant OTOF variant in Taiwanese families, accounting for approximately $70 \%$ of the total variants detected. This variant is specific in Taiwanese, and has not been reported in other populations, except in a Chinese series that reported a patient with heterozygous p.E1700Q [75]. In our previous study, we confirmed the founder effect of p.E1700Q in the Taiwanese population by haplotype analyses [76]. p.E1700Q is presumed to be disease-causing or disease-related because it is evolutionarily conserved among mammalian species and its pathogenicity is supported by multiple prediction programs (PolyPhen-2, MutationTaster, and MutationAssessor). However, we recently identified another OTOF variant that is in linkage disequilibrium with p.E1700Q and also likely to be pathogenic. Functional studies are underway to examine the pathogenicity of p.E1700Q.

\subsection{MYO15A Mutations}

MYO15A is a large gene composed of 67 exons; more than 190 MYO15A mutations have been documented to date [77]. The large size of the gene and the heterogeneity of the mutations have 
precluded the inclusion of MYO15A screening into routine deafness genetic examinations using conventional sequencing strategies [78-80]. However, recent advances in NGS technology have facilitated mutation detection and confirmed MYO15A mutations as a common cause of autosomal recessive non-syndromic hearing loss in several populations [81-83].

The phenotypes of MYO15A mutations vary from pre-lingual bilateral severe-to-profound SNHI $[81,84]$ to post-lingual progressive SNHI $[79,85]$, with residual hearing at lower frequencies occasionally noted $[79,81,83,86]$. In our cohort, approximately $80 \%$ of the patients with bi-allelic MYO15A mutations had severe to profound SNHI. We also found that patients with N-terminal domain mutations and those with non- $\mathrm{N}$ terminal domain mutations presented different hearing profiles, which was consistent with previous reports [86-88].

\subsection{MTRNR1 Mutations}

Approximate $2 \%$ of Taiwanese families with idiopathic SNHI were confirmed to have m.1555A $>$ G mutation in the MTRNR1 gene [39]. Large-scale genetic screenings in newborns revealed that m.1555A $>\mathrm{G}$ has a prevalence of approximately $1 / 1000$ in the general Taiwanese population [50,51,89], which is comparable to that in other populations [90-93]. Notably, all participating babies in our studies with m.1555A $>$ G passed newborn hearing screening at birth $[50,51,89]$. The identification of this mutation might thus have important clinical implications for these babies and their maternal relatives. Specifically, the use of aminoglycoside antibiotics should be avoided in these babies and their maternal relatives, as permanent profound SNHI might occur immediately after exposure to this medication [94,95].

\subsection{Mutations in Other Deafness Genes}

As most of the currently-available NGS diagnostic panels also include syndromic HHI genes, occasionally earlier diagnosis and earlier treatment could be facilitated in families with certain types of syndromic HHI. For instance, we previously identified a GATA3 c.149delT mutation using our NGS panel in a family which was originally considered to have autosomal dominant non-syndromic HHI. On the basis of the genetic finding, we could later ascertain the phenotypes of HDR syndrome in this family [34]. In other words, the genetic diagnosis of HDR syndrome was confirmed prior to clinical diagnosis in this family. This is important for this family, as a close follow-up of the parathyroid and renal functions is warranted for the affected family members.

\subsection{Strengths and Limitations of the Study}

The major strength of this study lies in the demonstration of the genetic epidemiology of HHI in a large Taiwanese cohort, in which the phenotypes and genotypes of the participants were ascertained at a single institute. However, some limitations of this study merit further discussion. First, although genetic diagnosis was confirmed in approximately half of our families, it is possible that some pathogenic variants, including structural variants, non-coding variants, and mosaic variants, have been missed due to current technical bottlenecks. Second, as targeted NGS examination was not available until the last few years of the study period, only a limited number of families in our longitudinal cohort had access to it. This might have led to selection or recruitment biases.

\section{Conclusions}

In conclusion, we presented comprehensive genetic results for a large Taiwanese cohort with idiopathic SNHI and demonstrated that NGS-based or NGS-assisted genetic examination strategies could achieve diagnosis in approximately half of the families. Common deafness-associated genes in Taiwanese families, in order of prevalence, included GJB2, SLC26A4, OTOF, MYO15A, and MTRNR1, which are similar to those found in other populations. However, Taiwanese patients possessed some unique mutations within these common genes. These findings may have important clinical implications 
for refining molecular diagnostics, facilitating genetic counseling, and enabling precision medicine for the management of HHI.

Supplementary Materials: The following are available online at http://www.mdpi.com/2073-4425/10/10/772/s1, Table S1: All NCBI Reference Sequences of other deafness genes.

Author Contributions: C.-C.W. designed and directed the project; C.-Y.T., Y.-H.L., P.-Y.C., P.-H.L. contributed equally to the data analyses and manuscript preparation; Y.-F.C., C.-M.W. and T.-C.L. provided clinical resources and advices; Y.-H.L. provided analysis techniques; C.-Y.L. reviewed previous literature; J.E. collaborated in sample collection and phenotype characterization; P.-L.C. and C.-J.H. supervised the whole research.

Funding: This study was supported by research grants from the Ministry of Science and Technology of Taiwan (MOST 107-2314-B-002 -137 -MY3 and MOST 107-2622-B-002 -008 -CC2).

Acknowledgments: We would like to thank all subjects and their family members for participating in the present study, and the Children Charity Association for the assistance in the recruitment of subjects. We also wish to thank National Core Facility for Biopharmaceuticals (NCFB, MOST 108-2319-B-492 -001) for support and National Center for High-performance Computing (NCHC) of National Applied Research Laboratories (NARLabs) in Taiwan for providing computational and storage resources.

Conflicts of Interest: The authors declare no conflict of interest.

\section{References}

1. Morton, C.C.; Nance, W.E. Newborn hearing screening-A silent revolution. N. Engl. J. Med. 2006, 354, 2151-2164. [CrossRef] [PubMed]

2. Wake, M.; Tobin, S.; Cone-Wesson, B.; Dahl, H.H.; Gillam, L.; McCormick, L.; Poulakis, Z.; Rickards, F.W.; Saunders, K.; Ukoumunne, O.C.; et al. Slight/mild sensorineural hearing loss in children. Pediatrics 2006, 118, 1842-1851. [CrossRef] [PubMed]

3. Feder, K.P.; Michaud, D.; McNamee, J.; Fitzpatrick, E.; Ramage-Morin, P.; Beauregard, Y. Prevalence of Hearing Loss Among a Representative Sample of Canadian Children and Adolescents, 3 to 19 Years of Age. Ear Hear. 2017, 38, 7-20. [CrossRef] [PubMed]

4. Hilgert, N.; Smith, R.J.; Van Camp, G. Forty-six genes causing nonsyndromic hearing impairment: Which ones should be analyzed in DNA diagnostics? Mutat. Res. 2009, 681, 189-196. [CrossRef] [PubMed]

5. Abe, S.; Usami, S.; Shinkawa, H.; Kelley, P.M.; Kimberling, W.J. Prevalent connexin 26 gene (GJB2) mutations in Japanese. J. Med. Genet. 2000, 37, 41-43. [CrossRef] [PubMed]

6. Hwa, H.L.; Ko, T.M.; Hsu, C.J.; Huang, C.H.; Chiang, Y.L.; Oong, J.L.; Chen, C.C.; Hsu, C.K. Mutation spectrum of the connexin 26 (GJB2) gene in Taiwanese patients with prelingual deafness. Genet. Med. 2003, 5, 161-165. [CrossRef]

7. Park, H.J.; Hahn, S.H.; Chun, Y.M.; Park, K.; Kim, H.N. Connexin26 mutations associated with nonsyndromic hearing loss. Laryngoscope 2000, 110, 1535-1538. [CrossRef] [PubMed]

8. Gurtler, N.; Kim, Y.; Mhatre, A.; Muller, R.; Probst, R.; Lalwani, A.K. GJB2 mutations in the Swiss hearing impaired. Ear Hear. 2003, 24, 440-447. [CrossRef]

9. Denoyelle, F.; Weil, D.; Maw, M.A.; Wilcox, S.A.; Lench, N.J.; Allen-Powell, D.R.; Osborn, A.H.; Dahl, H.H.; Middleton, A.; Houseman, M.J.; et al. Prelingual deafness: High prevalence of a 30delG mutation in the connexin 26 gene. Hum. Mol. Genet. 1997, 6, 2173-2177. [CrossRef]

10. Frei, K.; Szuhai, K.; Lucas, T.; Weipoltshammer, K.; Schofer, C.; Ramsebner, R.; Baumgartner, W.D.; Raap, A.K.; Bittner, R.; Wachtler, F.J.; et al. Connexin 26 mutations in cases of sensorineural deafness in eastern Austria. Eur. J. Hum. Genet. 2002, 10, 427-432. [CrossRef]

11. Stinckens, C.; Kremer, H.; van Wijk, E.; Hoefsloot, L.H.; Huygen, P.L.; Standaert, L.; Fryns, J.P.; Cremers, C.W. Longitudinal phenotypic analysis in patients with connexin 26 (GJB2) (DFNB1) and connexin 30 (GJB6) mutations. Ann. Otol. Rhinol. Laryngol. 2004, 113, 587-593. [CrossRef] [PubMed]

12. Morell, R.J.; Kim, H.J.; Hood, L.J.; Goforth, L.; Friderici, K.; Fisher, R.; Van Camp, G.; Berlin, C.I.; Oddoux, C.; Ostrer, H.; et al. Mutations in the connexin 26 gene (GJB2) among Ashkenazi Jews with nonsyndromic recessive deafness. N. Engl. J. Med. 1998, 339, 1500-1505. [CrossRef] [PubMed]

13. Sirmaci, A.; Akcayoz-Duman, D.; Tekin, M. The c.IVS1+1G>A mutation in the GJB2 gene is prevalent and large deletions involving the GJB6 gene are not present in the Turkish population. J. Genet. 2006, 85, $213-216$. [CrossRef] [PubMed] 
14. Mahdieh, N.; Nishimura, C.; Ali-Madadi, K.; Riazalhosseini, Y.; Yazdan, H.; Arzhangi, S.; Jalalvand, K.; Ebrahimi, A.; Kazemi, S.; Smith, R.J.; et al. The frequency of GJB2 mutations and the Delta (GJB6-D13S1830) deletion as a cause of autosomal recessive non-syndromic deafness in the Kurdish population. Clin. Genet. 2004, 65, 506-508. [CrossRef] [PubMed]

15. Bajaj, Y.; Sirimanna, T.; Albert, D.M.; Qadir, P.; Jenkins, L.; Bitner-Glindzicz, M. Spectrum of GJB2 mutations causing deafness in the British Bangladeshi population. Clin. Otolaryngol. 2008, 33, 313-318. [CrossRef] [PubMed]

16. Padma, G.; Ramchander, P.V.; Nandur, U.V.; Padma, T. GJB2 and GJB6 gene mutations found in Indian probands with congenital hearing impairment. J. Genet. 2009, 88, 267-272. [CrossRef]

17. Campbell, C.; Cucci, R.A.; Prasad, S.; Green, G.E.; Edeal, J.B.; Galer, C.E.; Karniski, L.P.; Sheffield, V.C.; Smith, R.J. Pendred syndrome, DFNB4, and PDS/SLC26A4 identification of eight novel mutations and possible genotype-phenotype correlations. Hum. Mutat. 2001, 17, 403-411. [CrossRef]

18. Coyle, B.; Reardon, W.; Herbrick, J.A.; Tsui, L.C.; Gausden, E.; Lee, J.; Coffey, R.; Grueters, A.; Grossman, A.; Phelps, P.D.; et al. Molecular analysis of the PDS gene in Pendred syndrome. Hum. Mol. Genet. 1998, 7, 1105-1112. [CrossRef]

19. Tsukamoto, K.; Suzuki, H.; Harada, D.; Namba, A.; Abe, S.; Usami, S. Distribution and frequencies of PDS (SLC26A4) mutations in Pendred syndrome and nonsyndromic hearing loss associated with enlarged vestibular aqueduct: A unique spectrum of mutations in Japanese. Eur. J. Hum. Genet. 2003, 11, 916-922. [CrossRef]

20. Park, H.J.; Lee, S.J.; Jin, H.S.; Lee, J.O.; Go, S.H.; Jang, H.S.; Moon, S.K.; Lee, S.C.; Chun, Y.M.; Lee, H.K.; et al. Genetic basis of hearing loss associated with enlarged vestibular aqueducts in Koreans. Clin. Genet. 2005, 67, 160-165. [CrossRef]

21. Dai, P.; Li, Q.; Huang, D.; Yuan, Y.; Kang, D.; Miller, D.T.; Shao, H.; Zhu, Q.; He, J.; Yu, F.; et al. SLC26A4 c.919-2A>G varies among Chinese ethnic groups as a cause of hearing loss. Genet. Med. Off. J. Am. Coll. Med Genet. 2008, 10, 586-592. [CrossRef]

22. Executive Yuan of R.O.C. Republic of China Yearbook; Executive Yuan of R.O.C.: Taipei, Taiwan, 2016; ISBN 9789860499490.

23. Chen, C.H.; Yang, J.H.; Chiang, C.W.K.; Hsiung, C.N.; Wu, P.E.; Chang, L.C.; Chu, H.W.; Chang, J.; Song, I.W.; Yang, S.L.; et al. Population structure of Han Chinese in the modern Taiwanese population based on 10,000 participants in the Taiwan Biobank project. Hum. Mol. Genet. 2016, 25, 5321-5331. [CrossRef] [PubMed]

24. Wu, C.C.; Lin, S.Y.; Su, Y.N.; Fang, M.Y.; Chen, S.U.; Hsu, C.J. Preimplantation genetic diagnosis (embryo screening) for enlarged vestibular aqueduct due to SLC26A4 mutation. Audiol. Neuro-Otol. 2010, 15, 311-317. [CrossRef] [PubMed]

25. Wu, C.C.; Hsu, C.J.; Huang, F.L.; Lin, Y.H.; Lin, Y.H.; Liu, T.C.; Wu, C.M. Timing of cochlear implantation in auditory neuropathy patients with OTOF mutations: Our experience with 10 patients. Clin. Otolaryngol. 2018, 43, 352-357. [CrossRef] [PubMed]

26. Lin, P.H.; Hsu, C.J.; Lin, Y.H.; Lin, Y.H.; Lee, H.Y.; Wu, C.C.; Liu, T.C. Etiologic and Audiologic Characteristics of Patients With Pediatric-Onset Unilateral and Asymmetric Sensorineural Hearing Loss. JAMA Otolaryngol. Head Neck Surg. 2017, 143, 912-919. [CrossRef]

27. Sennaroglu, L.; Saatci, I. A new classification for cochleovestibular malformations. Laryngoscope 2002, 112, 2230-2241. [CrossRef] [PubMed]

28. McClay, J.E.; Tandy, R.; Grundfast, K.; Choi, S.; Vezina, G.; Zalzal, G.; Willner, A. Major and minor temporal bone abnormalities in children with and without congenital sensorineural hearing loss. Arch. Otolaryngol. Head Neck Surg. 2002, 128, 664-671. [CrossRef]

29. Wu, C.C.; Chen, Y.S.; Chen, P.J.; Hsu, C.J. Common clinical features of children with enlarged vestibular aqueduct and Mondini dysplasia. Laryngoscope 2005, 115, 132-137. [CrossRef]

30. Wu, C.C.; Chen, P.J.; Chiu, Y.H.; Lu, Y.C.; Wu, M.C.; Hsu, C.J. Prospective mutation screening of three common deafness genes in a large Taiwanese cohort with idiopathic bilateral sensorineural hearing impairment reveals a difference in the results between families from hospitals and those from rehabilitation facilities. Audiol. Neurotol. 2008, 13, 172-181. [CrossRef] 
31. Wu, C.C.; Yeh, T.H.; Chen, P.J.; Hsu, C.J. Prevalent SLC26A4 mutations in patients with enlarged vestibular aqueduct and/or Mondini dysplasia: A unique spectrum of mutations in Taiwan, including a frequent founder mutation. Laryngoscope 2005, 115, 1060-1064. [CrossRef]

32. Wu, C.C.; Chen, P.J.; Hsu, C.J. Specificity of SLC26A4 mutations in the pathogenesis of inner ear malformations. Audiol. Neurootol. 2005, 10, 234-242. [CrossRef] [PubMed]

33. Wu, C.C.; Lin, Y.H.; Lu, Y.C.; Chen, P.J.; Yang, W.S.; Hsu, C.J.; Chen, P.L. Application of massively parallel sequencing to genetic diagnosis in multiplex families with idiopathic sensorineural hearing impairment. PLOS ONE 2013, 8, e57369. [CrossRef] [PubMed]

34. Lin, Y.H.; Wu, C.C.; Hsu, T.Y.; Chiu, W.Y.; Hsu, C.J.; Chen, P.L. Identification of a novel GATA3 mutation in a deaf Taiwanese family by massively parallel sequencing. Mutat. Res. 2015, 771, 1-5. [CrossRef] [PubMed]

35. Lin, Y.H.; Lin, Y.H.; Lu, Y.C.; Liu, T.C.; Chen, C.Y.; Hsu, C.J.; Chen, P.L.; Wu, C.C. A novel missense variant in the nuclear localization signal of POU4F3 causes autosomal dominant non-syndromic hearing loss. Sci. Rep. 2017, 7, 7551. [CrossRef]

36. Lin, Y.H.; Wu, C.C.; Lin, Y.H.; Lu, Y.C.; Chen, C.S.; Liu, T.C.; Chen, P.L.; Hsu, C.J. Targeted Next-Generation Sequencing Facilitates Genetic Diagnosis and Provides Novel Pathogenetic Insights into Deafness with Enlarged Vestibular Aqueduct. J. Mol. Diagn. 2019, 21, 138-148. [CrossRef] [PubMed]

37. Chen, P.Y.; Lin, Y.H.; Liu, T.C.; Lin, Y.H.; Tseng, L.H.; Yang, T.H.; Chen, P.L.; Wu, C.C.; Hsu, C.J. Prediction Model for Audiological Outcomes in Patients With GJB2 Mutations. Ear Hear. 2019. [CrossRef]

38. Wu, C.C.; Lu, Y.C.; Chen, P.J.; Yeh, P.L.; Su, Y.N.; Hwu, W.L.; Hsu, C.J. Phenotypic analyses and mutation screening of the SLC26A4 and FOXI1 genes in 101 Taiwanese families with bilateral nonsyndromic enlarged vestibular aqueduct (DFNB4) or Pendred syndrome. Audiol. Neurootol. 2010, 15, 57-66. [CrossRef]

39. Wu, C.C.; Chiu, Y.H.; Chen, P.J.; Hsu, C.J. Prevalence and clinical features of the mitochondrial m.1555A $>$ G mutation in Taiwanese patients with idiopathic sensorineural hearing loss and association of haplogroup $\mathrm{F}$ with low penetrance in three families. Ear Hear. 2007, 28, 332-342. [CrossRef]

40. Zhao, H.; Li, R.H.; Wang, Q.J.; Yan, Q.F.; Deng, J.H.; Han, D.Y.; Bai, Y.D.; Young, W.Y.; Guan, M.X. Maternally inherited aminoglycoside-induced and nonsyndromic deafness is associated with the novel C1494T mutation in the mitochondrial 12S rRNA gene in a large chinese family. Am. J. Hum. Genet. 2004, 74, 139-152. [CrossRef]

41. Moassass, F.; Al-Halabi, B.; Nweder, M.S.; Al-Achkar, W. Investigation of the mtDNA mutations in Syrian families with non-syndromic sensorineural hearing loss. Int. J. Pediatr. Otorhi. 2018, 113, 110-114. [CrossRef]

42. Su, C.C.; Yang, J.J.; Shieh, J.C.; Su, M.C.; Li, S.Y. Identification of novel mutations in the KCNQ4 gene of patients with nonsyndromic deafness from Taiwan. Audiol. Neuro-Otol. 2007, 12, 20-26. [CrossRef] [PubMed]

43. Su, M.C.; Yang, J.J.; Su, C.C.; Hsin, C.H.; Li, S.Y. Identification of novel variants in the Myosin VIIA gene of patients with nonsyndromic hearing loss from Taiwan. Int. J. Pediatr. Otorhinolaryngol. 2009, 73, 811-815. [CrossRef] [PubMed]

44. Wang, S.H.; Wu, C.C.; Lu, Y.C.; Lin, Y.H.; Su, Y.N.; Hwu, W.L.; Yu, I.S.; Hsu, C.J. Mutation screening of the EYA1, SIX1, and SIX5 genes in an east asian cohort with branchio-oto-renal syndrome. Laryngoscope 2012, 122, 1130-1136. [CrossRef] [PubMed]

45. Shearer, A.E.; DeLuca, A.P.; Hildebrand, M.S.; Taylor, K.R.; Gurrola, J.; Scherer, S.; Scheetz, T.E.; Smith, R.J.H. Comprehensive genetic testing for hereditary hearing loss using massively parallel sequencing. Proc. Natl. Acad. Sci. USA 2010, 107, 21104-21109. [CrossRef] [PubMed]

46. Brownstein, Z.; Friedman, L.M.; Shahin, H.; Oron-Karni, V.; Kol, N.; Abu Rayyan, A.; Parzefall, T.; Lev, D.; Shalev, S.; Frydman, M.; et al. Targeted genomic capture and massively parallel sequencing to identify genes for hereditary hearing loss in Middle Eastern families. Genome Biol. 2011, 12, R89. [CrossRef] [PubMed]

47. Miyagawa, M.; Naito, T.; Nishio, S.Y.; Kamatani, N.; Usami, S. Targeted exon sequencing successfully discovers rare causative genes and clarifies the molecular epidemiology of Japanese deafness patients. PLOS ONE 2013, 8, e71381. [CrossRef]

48. Sommen, M.; Schrauwen, I.; Vandeweyer, G.; Boeckx, N.; Corneveaux, J.J.; van den Ende, J.; Boudewyns, A.; De Leenheer, E.; Janssens, S.; Claes, K.; et al. DNA Diagnostics of Hereditary Hearing Loss: A Targeted Resequencing Approach Combined with a Mutation Classification System. Hum. Mutat. 2016, 37, 812-819. [CrossRef] 
49. Sun, Y.; Yuan, J.; Wu, L.; Li, M.; Cui, X.; Yan, C.; Du, L.; Mao, L.; Man, J.; Li, W.; et al. Panel-based NGS reveals disease-causing mutations in hearing loss patients using BGISEQ-500 platform. Medicine 2019, 98, e14860. [CrossRef]

50. Lu, C.Y.; Tsao, P.N.; Ke, Y.Y.; Lin, Y.H.; Lin, Y.H.; Hung, C.C.; Su, Y.N.; Hsu, W.C.; Hsieh, W.S.; Huang, L.M.; et al. Concurrent Hearing, Genetic, and Cytomegalovirus Screening in Newborns, Taiwan. J. Pediatr. 2018, 199, 144-150. [CrossRef]

51. Wu, C.C.; Tsai, C.H.; Hung, C.C.; Lin, Y.H.; Lin, Y.H.; Huang, F.L.; Tsao, P.N.; Su, Y.N.; Lee, Y.L.; Hsieh, W.S.; et al. Newborn genetic screening for hearing impairment: A population-based longitudinal study. Genet. Med. 2017, 19, 6-12. [CrossRef]

52. Li, L.; Lu, J.; Tao, Z.; Huang, Q.; Chai, Y.; Li, X.; Huang, Z.; Li, Y.; Xiang, M.; Yang, J.; et al. The p.V37I exclusive genotype of GJB2: A genetic risk-indicator of postnatal permanent childhood hearing impairment. PLOS ONE 2012, 7, e36621. [CrossRef] [PubMed]

53. Wattanasirichaigoon, D.; Limwongse, C.; Jariengprasert, C.; Yenchitsomanus, P.T.; Tocharoenthanaphol, C.; Thongnoppakhun, W.; Thawil, C.; Charoenpipop, D.; Pho-iam, T.; Thongpradit, S.; et al. High prevalence of V37I genetic variant in the connexin-26 (GJB2) gene among non-syndromic hearing-impaired and control Thai individuals. Clin. Genet. 2004, 66, 452-460. [CrossRef] [PubMed]

54. Erdenechuluun, J.; Lin, Y.H.; Ganbat, K.; Bataakhuu, D.; Makhbal, Z.; Tsai, C.Y.; Lin, Y.H.; Chan, Y.H.; Hsu, C.J.; Hsu, W.C.; et al. Unique spectra of deafness-associated mutations in Mongolians provide insights into the genetic relationships among Eurasian populations. PLoS ONE 2018, 13, e0209797. [CrossRef] [PubMed]

55. Snoeckx, R.L.; Huygen, P.L.; Feldmann, D.; Marlin, S.; Denoyelle, F.; Waligora, J.; Mueller-Malesinska, M.; Pollak, A.; Ploski, R.; Murgia, A.; et al. GJB2 mutations and degree of hearing loss: A multicenter study. Am. J. Hum. Genet. 2005, 77, 945-957. [CrossRef] [PubMed]

56. Oguchi, T.; Ohtsuka, A.; Hashimoto, S.; Oshima, A.; Abe, S.; Kobayashi, Y.; Nagai, K.; Matsunaga, T.; Iwasaki, S.; Nakagawa, T.; et al. Clinical features of patients with GJB2 (connexin 26) mutations: Severity of hearing loss is correlated with genotypes and protein expression patterns. J. Hum. Genet. 2005, 50, 76-83. [CrossRef] [PubMed]

57. Chan, D.K.; Schrijver, I.; Chang, K.W. Connexin-26-associated deafness: Phenotypic variability and progression of hearing loss. Genet. Med. 2010, 12, 174-181. [CrossRef]

58. Kenna, M.A.; Feldman, H.A.; Neault, M.W.; Frangulov, A.; Wu, B.L.; Fligor, B.; Rehm, H.L. Audiologic phenotype and progression in GJB2 (Connexin 26) hearing loss. Arch. Otolaryngol. Head Neck Surg. 2010, 136, 81-87. [CrossRef]

59. Tsukada, K.; Nishio, S.; Usami, S. A large cohort study of GJB2 mutations in Japanese hearing loss patients. Clin. Genet. 2010, 78, 464-470. [CrossRef] [PubMed]

60. Azaiez, H.; Booth, K.T.; Ephraim, S.S.; Crone, B.; Black-Ziegelbein, E.A.; Marini, R.J.; Shearer, A.E.; Sloan-Heggen, C.M.; Kolbe, D.; Casavant, T.; et al. Genomic Landscape and Mutational Signatures of Deafness-Associated Genes. Am. J. Hum. Genet. 2018, 103, 484-497. [CrossRef]

61. Tsukada, K.; Nishio, S.; Hattori, M.; Usami, S. Ethnic-Specific Spectrum of GJB2 and SLC26A4 Mutations: Their Origin and a Literature Review. Ann. Oto. Rhinol. Laryngol. 2015, 124, 61s-76s. [CrossRef]

62. Song, M.J.; Lee, S.T.; Lee, M.K.; Ji, Y.; Kim, J.W.; Ki, C.S. Estimation of carrier frequencies of six autosomal-recessive Mendelian disorders in the Korean population. J. Hum. Genet. 2012, 57, 139-144. [CrossRef] [PubMed]

63. Nishio, S.Y.; Usami, S. Deafness gene variations in a 1120 nonsyndromic hearing loss cohort: Molecular epidemiology and deafness mutation spectrum of patients in Japan. Ann. Otol. Rhinol. Laryngol. 2015, 124, 49S-60S. [CrossRef] [PubMed]

64. Shearer, A.E.; Kolbe, D.L.; Azaiez, H.; Sloan, C.M.; Frees, K.L.; Weaver, A.E.; Clark, E.T.; Nishimura, C.J.; Black-Ziegelbein, E.A.; Smith, R.J.H. Copy number variants are a common cause of non-syndromic hearing loss. Genome Med. 2014, 6. [CrossRef] [PubMed]

65. Pique, L.M.; Brennan, M.L.; Davidson, C.J.; Schaefer, F.; Greinwald, J.; Schrijver, I. Mutation analysis of the SLC26A4, FOXI1 and KCNJ10 genes in individuals with congenital hearing loss. Peerj 2014, 2. [CrossRef] [PubMed] 
66. Rendtorff, N.D.; Schrijver, I.; Lodahl, M.; Rodriguez-Paris, J.; Johnsen, T.; Hansen, E.C.; Nickelsen, L.A.A.; Tumer, Z.; Fagerheim, T.; Wetke, R.; et al. SLC26A4 mutation frequency and spectrum in 109 Danish Pendred syndrome/DFNB4 probands and a report of nine novel mutations. Clin. Genet. 2013, 84, 388-391. [CrossRef] [PubMed]

67. Miyagawa, M.; Nishio, S.Y.; Usami, S.; Deafness Gene Study, C. Mutation spectrum and genotype-phenotype correlation of hearing loss patients caused by SLC26A4 mutations in the Japanese: A large cohort study. J. Hum. Genet. 2014, 59, 262-268. [CrossRef]

68. Wang, Q.J.; Zhao, Y.L.; Rao, S.Q.; Guo, Y.F.; Yuan, H.; Zong, L.; Guan, J.; Xu, B.C.; Wang, D.Y.; Han, M.K.; et al. A distinct spectrum of SLC26A4 mutations in patients with enlarged vestibular aqueduct in China. Clin. Genet. 2007, 72, 245-254. [CrossRef]

69. Pang, X.H.; Chai, Y.C.; Chen, P.H.; He, L.X.; Wang, X.W.; Wu, H.; Yang, T. Mono-allelic mutations of SLC26A4 is over-presented in deaf patients with non-syndromic enlarged vestibular aqueduct. Int. J. Pediatr. Otorhinolaryngol. 2015, 79, 1351-1353. [CrossRef]

70. Albert, S.; Blons, H.; Jonard, L.; Feldmann, D.; Chauvin, P.; Loundon, N.; Sergent-Allaoui, A.; Houang, M.; Joannard, A.; Schmerber, S.; et al. SLC26A4 gene is frequently involved in nonsyndromic hearing impairment with enlarged vestibular aqueduct in Caucasian populations. Eur. J. Hum. Genet. 2006, 14, 773-779. [CrossRef]

71. Azaiez, H.; Yang, T.; Prasad, S.; Sorensen, J.L.; Nishimura, C.J.; Kimberling, W.J.; Smith, R.J. Genotype-phenotype correlations for SLC26A4-related deafness. Hum. Genet. 2007. [CrossRef]

72. Pryor, S.P.; Madeo, A.C.; Reynolds, J.C.; Sarlis, N.J.; Arnos, K.S.; Nance, W.E.; Yang, Y.; Zalewski, C.K.; Brewer, C.C.; Butman, J.A.; et al. SLC26A4/PDS genotype-phenotype correlation in hearing loss with enlargement of the vestibular aqueduct (EVA): Evidence that Pendred syndrome and non-syndromic EVA are distinct clinical and genetic entities. J. Med. Genet. 2005, 42, 159-165. [CrossRef] [PubMed]

73. Manchaiah, V.K.; Zhao, F.; Danesh, A.A.; Duprey, R. The genetic basis of auditory neuropathy spectrum disorder (ANSD). Int. J. Pediatr. Otorhinolaryngol. 2011, 75, 151-158. [CrossRef] [PubMed]

74. Moser, T.; Starr, A. Auditory neuropathy-Neural and synaptic mechanisms. Nat. Rev. Neurol. 2016, 12, 135-149. [CrossRef] [PubMed]

75. Chen, K.; Liu, M.; Wu, X.; Zong, L.; Jiang, H. Targeted next generation sequencing reveals OTOF mutations in auditory neuropathy spectrum disorder. Int. J. Pediatr. Otorhinolaryngol. 2018, 115, 19-23. [CrossRef]

76. Chiu, Y.H.; Wu, C.C.; Lu, Y.C.; Chen, P.J.; Lee, W.Y.; Liu, A.Y.; Hsu, C.J. Mutations in the OTOF gene in Taiwanese patients with auditory neuropathy. Audiol. Neurootol. 2010, 15, 364-374. [CrossRef] [PubMed]

77. Rehman, A.U.; Bird, J.E.; Faridi, R.; Shahzad, M.; Shah, S.; Lee, K.; Khan, S.N.; Imtiaz, A.; Ahmed, Z.M.; Riazuddin, S.; et al. Mutational Spectrum of MYO15A and the Molecular Mechanisms of DFNB3 Human Deafness. Hum. Mutat. 2016, 37, 991-1003. [CrossRef]

78. Motavaf, M.; Soveizi, M.; Maleki, M.; Mahdieh, N. MYO15A splicing mutations in hearing loss: A review literature and report of a novel mutation. Int. J. Pediatr. Otorhinolaryngol. 2017, 96, 35-38. [CrossRef]

79. Miyagawa, M.; Nishio, S.Y.; Hattori, M.; Moteki, H.; Kobayashi, Y.; Sato, H.; Watanabe, T.; Naito, Y.; Oshikawa, C.; Usami, S. Mutations in the MYO15A gene are a significant cause of nonsyndromic hearing loss: Massively parallel DNA sequencing-based analysis. Ann. Otol. Rhinol. Laryngol. 2015, 124, 158s-168s. [CrossRef]

80. Usami, S.; Nishio, S.Y.; Nagano, M.; Abe, S.; Yamaguchi, T. Simultaneous screening of multiple mutations by invader assay improves molecular diagnosis of hereditary hearing loss: A multicenter study. PLoS ONE 2012, 7, e31276. [CrossRef]

81. Bashir, R.; Fatima, A.; Naz, S. Prioritized sequencing of the second exon of MYO15A reveals a new mutation segregating in a Pakistani family with moderate to severe hearing loss. Eur. J. Med Genet. 2012, 55, 99-102. [CrossRef]

82. Bai, X.; Nian, S.; Feng, L.; Ruan, Q.; Luo, X.; Wu, M.; Yan, Z. Identification of novel variants in MYO15A, OTOF, and RDX with hearing loss by next-generation sequencing. Mol. Genet. Genom. Med. 2019. [CrossRef] [PubMed]

83. Fattahi, Z.; Shearer, A.E.; Babanejad, M.; Bazazzadegan, N.; Almadani, S.N.; Nikzat, N.; Jalalvand, K.; Arzhangi, S.; Esteghamat, F.; Abtahi, R.; et al. Screening for MYO15A gene mutations in autosomal recessive nonsyndromic, GJB2 negative Iranian deaf population. Am. J. Med Genet. Part A 2012, 158, 1857-1864. [CrossRef] [PubMed] 
84. Woo, H.M.; Park, H.J.; Baek, J.I.; Park, M.H.; Kim, U.K.; Sagong, B.; Koo, S.K. Whole-exome sequencing identifies MYO15A mutations as a cause of autosomal recessive nonsyndromic hearing loss in Korean families. BMC Med Genet. 2013, 14, 72. [CrossRef] [PubMed]

85. Chang, M.Y.; Lee, C.; Han, J.H.; Kim, M.Y.; Park, H.R.; Kim, N.; Park, W.Y.; Oh, D.Y.; Choi, B.Y. Expansion of phenotypic spectrum of MYO15A pathogenic variants to include postlingual onset of progressive partial deafness. BMC Med Genet. 2018, 19, 29. [CrossRef] [PubMed]

86. Chang, M.Y.; Kim, A.R.; Kim, N.K.; Lee, C.; Lee, K.Y.; Jeon, W.S.; Koo, J.W.; Oh, S.H.; Park, W.Y.; Kim, D.; et al. Identification and Clinical Implications of Novel MYO15A Mutations in a Non-consanguineous Korean Family by Targeted Exome Sequencing. Mol. Cells 2015, 38, 781-788. [CrossRef] [PubMed]

87. Zhang, F.; Xu, L.; Xiao, Y.; Li, J.; Bai, X.; Wang, H. Three MYO15A Mutations Identified in One Chinese Family with Autosomal Recessive Nonsyndromic Hearing Loss. Neural Plast. 2018, 2018, 5898025. [CrossRef] [PubMed]

88. Cengiz, F.B.; Duman, D.; Sirmaci, A.; Tokgoz-Yilmaz, S.; Erbek, S.; Ozturkmen-Akay, H.; Incesulu, A.; Edwards, Y.J.; Ozdag, H.; Liu, X.Z.; et al. Recurrent and private MYO15A mutations are associated with deafness in the Turkish population. Genet. Test. Mol. Biomark. 2010, 14, 543-550. [CrossRef] [PubMed]

89. Wu, C.C.; Hung, C.C.; Lin, S.Y.; Hsieh, W.S.; Tsao, P.N.; Lee, C.N.; Su, Y.N.; Hsu, C.J. Newborn Genetic Screening for Hearing Impairment: A Preliminary Study at a Tertiary Center. PLoS ONE 2011, 6. [CrossRef]

90. Bitner-Glindzicz, M.; Pembrey, M.; Duncan, A.; Heron, J.; Ring, S.M.; Hall, A.; Rahman, S. Prevalence of mitochondrial 1555A $\rightarrow$ G mutation in European children. N. Engl. J. Med. 2009, 360, 640-642. [CrossRef] [PubMed]

91. Igumnova, V.; Veidemane, L.; Viksna, A.; Capligina, V.; Zole, E.; Ranka, R. The prevalence of mitochondrial mutations associated with aminoglycoside-induced deafness in ethnic Latvian population: The appraisal of the evidence. J. Hum. Genet. 2019, 64, 199-206. [CrossRef]

92. Yao, G.D.; Li, S.X.; Chen, D.L.; Feng, H.Q.; Zhao, S.B.; Liu, Y.J.; Guo, L.L.; Yang, Z.M.; Zhang, X.F.; Sun, C.X.; et al. Combination of hearing screening and genetic screening for deafness-susceptibility genes in newborns. Exp. Ther. Med. 2014, 7, 218-222. [CrossRef] [PubMed]

93. Soini, H.K.; Karjalainen, M.K.; Hinttala, R.; Rautio, A.; Hallman, M.; Uusimaa, J. Mitochondrial hearing loss mutations among Finnish preterm and term-born infants. Audiol. Res. 2017, 7, 189. [CrossRef] [PubMed]

94. Gopel, W.; Berkowski, S.; Preuss, M.; Ziegler, A.; Kuster, H.; Felderhoff-Muser, U.; Gortner, L.; Mogel, M.; Hartel, C.; Herting, E.; et al. Mitochondrial mutation m.1555A > G as a risk factor for failed newborn hearing screening in a large cohort of preterm infants. BMC Pediatr. 2014, 14. [CrossRef] [PubMed]

95. Bindu, L.H.; Reddy, P.P. Genetics of aminoglycoside-induced and prelingual non-syndromic mitochondrial hearing impairment: A review. Int. J. Audiol. 2008, 47, 702-707. [CrossRef] [PubMed] 